\title{
Effect of a nutrient-enriched drink on energy intake and postprandial metabolism in overweight subjects
}

\author{
N.M. Al-Mana and M.D. Robertson \\ Department of Nutritional Sciences, Faculty of Health and Medical Sciences, University of Surrey, Guildford, Surrey, \\ GU2 $7 W G$
}

Viscous fibres, including guar gum, as well as calcium have been linked to weight management ${ }^{(1,2)}$. It has been suggested that appetite and food intake may be affected by calcium or /and dietary fibre intakes in the diet ${ }^{(2-4)}$. However, the impact of these as isolated food ingredients is unknown. The aim of the present study is to investigate the short-term effects of viscous fibre from fenugreek (Fen), and calcium $(\mathrm{Ca})$, on appetite, satiety and postprandial metabolism in overweight/obese females.

In this randomised single-blind balanced crossover study, ten healthy female overweight /obese subjects (aged 18-45 years, BMI 25$37 \mathrm{~kg} / \mathrm{m}^{2}$ ) participated. Subjects were required to consume a test breakfast, containing an enriched drink of viscous fibre from fenugreek (Fen) and calcium (Ca) or a fibre and calcium-free placebo (PL). Appetite sensations were assessed using visual analogue scales (VAS) every $30 \mathrm{~min}$ for 3 hours, postprandial metabolism concentrations, energy intakes from an ad libitum lunch and over 24 hours were assessed.

The Fen-ca enriched drink significantly reduced hunger $(p=0.006)$. This finding was combined with a reduction in energy intake at the ad libitum lunch (976 (SEM 27.64) kcal versus 1122 (SEM 66.98) kcal, respectively, $(p=0.030)$. However, no significant differences over $24 \mathrm{~h}$ were observed between treatment groups. The enriched drink had no significant effects on plasma insulin or GLP-1 concentrations. Acute consumption of viscous fibre and $\mathrm{Ca}$ reduced food intake, indicating an effect on short-term satiety, however the effects were limited to the acute period only and this reduced energy intake was compensated for later in the day at subsequent meals.

1. Zemel M, Shi H, Greer B et al. (2000) Regulation of adiposity by dietary calcium. Faseb $J$ 14, 1132-8.

2. Mathern JR, Raatz SK, Thomas W, et al. (2009) Effect of fenugreek fiber on satiety, blood glucose and insulin response and energy intake in obese subjects. Phyther Res 23, 1543-8.

3. Chaput J-P, Leblanc C, Pérusse L et al. (2014) Risk factors for adult overweight and obesity in the Quebec Family Study: have we been barking up the wrong tree? Obesity 17, 1964-70.

4. Davies KM, Heaney RP, Recker RR et al. (2000) Calcium Intake and Body Weight. J Clin Endocrinol Metab 85, 4635-8. 